\title{
AVERAGING SPECIAL VALUES OF DIRICHLET L-SERIES.
}

\author{
KEVIN JAMES
}

ABSTRACT. In this paper we derive estimates for weighted averages of the special values of Dirichlet $L$-series which generalize similar estimates of David and Pappalardi [1].

\section{INTRODUCTION.}

Fix $r, m, n \in \mathbb{Z}$ with $(m, n)=1$. Let $d_{p}(f)=\frac{r^{2}-4 p}{f^{2}}$ and $B(r)=\max \left(5, r^{2} / 4\right)$. Define

$$
S_{f}^{r}(m, n, X):=\left\{\begin{array}{c}
B(r)<p \leq X: \mathrm{p} \text { is prime; } p \equiv m \quad(\bmod n) ; \\
4 p \equiv r^{2} \quad\left(\bmod f^{2}\right) ; d_{p}(f) \equiv 0,1 \quad(\bmod 4)
\end{array}\right\}
$$

and

$$
A(r, m, n, X):=\sum_{f \leq 2 \sqrt{X}} \frac{1}{f} \sum_{p \in S_{f}^{r}(m, n, X)} L\left(1, \chi_{d_{p}(f)}\right) \log p
$$

David and Pappalardi (see [1] Theorem 3.1 and Lemma 4.1) proved an estimate for $A(r, 1,1, X)$ which was an integral part of their proof that the Lang-Trotter conjecture is true on average. In related work on the Lang-Trotter conjecture for elliptic curves with nontrivial rational torsion subgroups (see for example [2]) it was neccessary to prove similar estimates on $A(r, r-1, n, X)$ for various squarefree $n$. In this paper we give an estimate for $A(r, m, n, X)$ for $(m, n)=1$ arbitrary. In order to state the main result, we will need a bit more notation. We will let $\Delta^{r, m}=r^{2}-4 m$ and put

$$
\begin{aligned}
& \mathfrak{Q}_{r, m, n}^{<}=\left\{q>2, \text { prime }: q \mid n ; q \backslash X r ; \operatorname{ord}_{q}\left(\Delta^{r, m}\right)<\operatorname{ord}_{q}(n)\right\} \quad \text { and } \\
& \mathfrak{Q}_{r, m, n}^{\geq}=\left\{q>2, \operatorname{prime}: q \mid n ; q \not X r ; \operatorname{ord}_{q}\left(\Delta^{r, m}\right) \geq \operatorname{ord}_{q}(n)\right\}
\end{aligned}
$$

For $q \in \mathfrak{Q}_{r, m, n}^{<}$, we will denote by $\gamma_{q}$, the greatest integer which is less than $\operatorname{ord}_{q}\left(\Delta^{r, m}\right) / 2$, that is $\gamma_{q}:=\left\lfloor\left(\operatorname{ord}_{q}\left(\Delta^{r, m}\right)-1\right) / 2\right\rfloor$. Also, we will let

$$
\Gamma_{q}= \begin{cases}\left(\frac{\left(\Delta^{r, m}\right) / q^{\text {ord } q\left(\Delta^{r, m}\right)}}{q}\right) & \text { if } \operatorname{ord}_{q}\left(\Delta^{r, m}\right) \text { is even, positive and finite, } \\ 0 & \text { otherwise. }\end{cases}
$$

In this paper we prove:

Date: August 23, 2005.

2000 Mathematics Subject Classification. Primary (11M06);Secondary (11G05).

$K e y$ words and phrases. Dirichlet $L$-series, Special values of $L$-series.

The author is partially supported by NSF grant DMS-0090117. 


\section{Theorem 1.1.}

$$
A(r, m, n, X) \sim C_{r, m, n} X,
$$

where

$$
\begin{aligned}
& C_{r, m, n}=\frac{1}{\phi(n)} C_{r, m, n}(2) \prod_{\substack{q, \text { odd } \\
q \nmid n \\
q \nmid r}} \frac{q\left(q^{2}-q-1\right)}{(q+1)(q-1)^{2}} \prod_{\substack{q, \text { odd } \\
q \nmid n \\
q \mid r}} \frac{q^{2}}{q^{2}-1} \prod_{\substack{q|n \\
q| r}}\left(\frac{q\left(q+\left(\frac{-m}{q}\right)\right)}{q^{2}-1}\right) . \\
& \prod_{q \in \mathfrak{Q}_{r, m, n}^{<}}\left(1+\frac{q\left(\frac{\Delta^{r, m}}{q}\right)+\left(\frac{\Delta^{r, m}}{q}\right)^{2}+\frac{1}{q^{\operatorname{ord} q\left(\Delta^{r, m}\right) / 2}}\left(q \Gamma_{q}+q^{2} \Gamma_{q}^{2}\right)}{q^{2}-1}+\frac{\Gamma_{q}^{2}\left(q^{\left\lfloor\frac{\operatorname{ord} q\left(\Delta^{r, m}\right)-1}{2}\right\rfloor}-1\right)}{q^{\left\lfloor\frac{\operatorname{ord} q\left(\Delta^{r, m}\right)-1}{2}\right\rfloor}(q-1)}\right) . \\
& \prod_{q \in \mathfrak{Q}_{r, m, n}^{\geq}}\left(\frac{q^{\left\lfloor\frac{\operatorname{\operatorname {ord}(n)+1}}{2}\right\rfloor}-1}{q^{\left\lfloor\frac{\operatorname{ord} q(n)-1}{2}\right\rfloor}(q-1)}+\frac{q^{\operatorname{ord}_{q}(n)+2}}{q^{3\left\lfloor\frac{\operatorname{ord} q(n)+1}{2}\right\rfloor}\left(q^{2}-1\right)}\right)
\end{aligned}
$$

and $C_{r, m, n}(2)$ is defined by

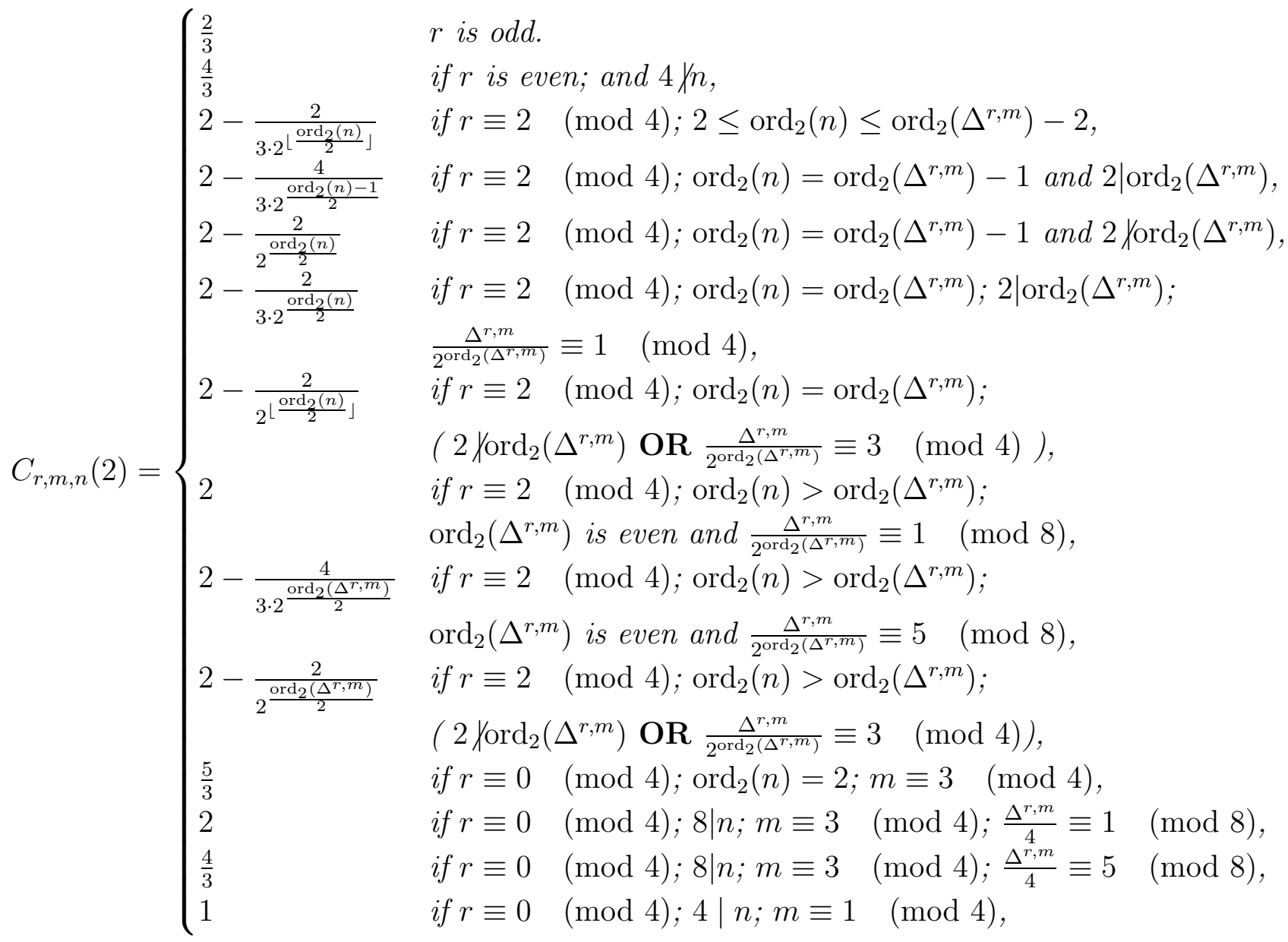




\section{Proofs.}

We first state the following result which is essentially due to David and Pappalardi, in the sense that one can obain a proof by following the same line of argument given in the proof of Theorem 3.1 in [1] with minor modifications such as carrying the condition $p \equiv m(\bmod n)$ throughout their argument.

Proposition 2.1. Suppose that $r, m, n \in \mathbb{Z}$ and that $(m, n)=1$. Then for any $c>0$,

$$
A(r, m, n, X)=K_{r, m, n} X+\mathrm{O}\left(\frac{X}{\log ^{c} X}\right)
$$

where

$$
K_{r, m, n}=\sum_{f=1}^{\infty} \frac{1}{f} \sum_{k=1}^{\infty} \frac{c_{f}^{r, m, n}(k)}{k \phi\left(\left[n ; k f^{2}\right]\right)}
$$

and

$$
c_{f}^{r, m, n}(k):=\sum_{\substack{a=(\bmod 4 k) \\ a \equiv 0,1)(\bmod 4) \\\left(r^{2}-a f^{2}, 4 k f^{2}\right)=4 \\ 4 m \equiv r^{2}-a f^{2}\left(\bmod \left(4 n, 4 k f^{2}\right)\right)}}\left(\frac{a}{k}\right) .
$$

For the sake of brevity, we omit the proof of this result and refer the reader to [1].

The proof of the main result now requires only a reconciling of the constants $K_{r, m, n}$ and $C_{r, m, n}$. To that end we begin with an investigation of the $c_{f}^{r, m, n}(k)$. For convenience, we will split these into two sums:

$$
c_{f, 0}^{r, m, n}(k):=\sum_{\substack{a \\ a=0(\bmod 4 k) \\\left(r^{2}-a f^{2}, 4 k f^{2}\right)=4 \\ 4 m \equiv r^{2}-a f^{2}\left(\bmod \left(4 n, 4 k f^{2}\right)\right)}}\left(\frac{a}{k}\right) \quad \text { and } \quad c_{f, 1}^{r, m, n}(k):=\sum_{\substack{a(\bmod 4 k) \\ a \equiv 1(\bmod 4) \\\left(r^{2}-a f^{2}, 4 k f^{2}\right)=4 \\ 4 m \equiv r^{2}-a f^{2}\left(\bmod \left(4 n, 4 k f^{2}\right)\right)}}\left(\frac{a}{k}\right) .
$$

In order to describe the behavior of the $c_{f, i}^{r, m, n}(k)$ 's we have the following lemmas. The first lemma follows directly from the above definitions. We state it for the sake of convenience only.

\section{Lemma 2.1.}

(1) For $c_{f, 0}^{r, m, n}(k)$ to be nonzero, it is necessary that we have $r$, even; $k$, odd, $(r / 2, f)=1$ and $\left(n, f^{2}\right) \mid\left((r / 2)^{2}-m\right)$.

(2) For $c_{f, 1}^{r, m, n}(k)$ to be nonzero, one of the following must hold.

(a) $r$ and $f$ are both odd, $(r, f)=1$ and $\left(n, f^{2}\right) \mid\left(\Delta^{r, m}\right)$.

(b) $r \equiv 2(\bmod 4),(r / 2, f)=1,\left(4 n, f^{2}\right) \mid\left(\Delta^{r, m}\right)$.

- If $\operatorname{ord}_{2}(n) \leq \operatorname{ord}_{2}\left(\Delta^{r, m}\right)-2$, then we require that $\operatorname{ord}_{2}\left(f^{2}\right) \geq \max \left(\operatorname{ord}_{2}(n)+2,4\right)$.

- If $\operatorname{ord}_{2}(n)=\operatorname{ord}_{2}\left(\Delta^{r, m}\right)-1$, then we require that $\operatorname{ord}_{2}\left(f^{2}\right)=\operatorname{ord}_{2}(n)+1$.

- If $\operatorname{ord}_{2}(n) \geq \operatorname{ord}_{2}\left(\Delta^{r, m}\right)$, then we require that $\operatorname{ord}_{2}\left(f^{2}\right)=\operatorname{ord}_{2}\left(\Delta^{r, m}\right)$ and $\frac{\Delta^{r, m}}{2^{\operatorname{ord}_{2}\left(\Delta^{r, m}\right)}} \equiv$ $1(\bmod 4)$. 
(c) $r \equiv 0(\bmod 4), f \equiv 2(\bmod 4),(r, f / 2)=1$ and $\left(n,(f / 2)^{2}\right) \mid\left((r / 2)^{2}-m\right)$. If $n \equiv 0$ $(\bmod 4)$, then we also need $m \equiv 3(\bmod 4)$.

Lemma 2.2. $c_{f, i}^{r, m, n}(k)(i=0,1)$ is a multiplicative function of $k$.

Proof. If $r$ is odd, $c_{f, 0}^{r, m, n}(k)=0$ and the multiplicativity of $c_{f, 1}^{r, m, n}(k)$ can be shown as in [1], lemma 3.3. So, we will consider only the case when $r$ is even.

In this case, if $(r / 2, f)=1,\left(n, f^{2}\right) \mid\left((r / 2)^{2}-m\right)$ and $k$ is odd, then we obtain

$$
c_{f, 0}^{r, m, n}(k)=\sum_{\substack{a \\\left((r / 2)^{2}-a f^{2}, k\right)=1}}^{\frac{(\bmod k)^{2}-m}{\left(n, f^{2}\right)} \equiv a \frac{f^{2}}{\left(n, f^{2}\right)}\left(\bmod \left(\frac{n}{\left(n, f^{2}\right)}, k\right)\right)}
$$

and zero otherwise. Since, $a$ runs through certain congruence classes modulo $k$ in the above sum, the multiplicativity of $c_{f, 0}^{r, m, n}(k)$ now follows form the Chinese remainder theorem and the multiplicative properties of the Legendre symbol.

We need only treat the cases in which $c_{f, 1}^{r, m, n}(k)$ is possibly nonzero (see lemma 2.1). For case 2a, if $k$ is odd, then we have

$$
c_{f, 1}^{r, m, n}(k)=\sum_{\substack{a \in \mathbb{Z} / k \mathbb{Z} \\\left(r^{2}-a f^{2}, k\right)=1}}\left(\frac{a}{k}\right) .
$$

In cases $2 \mathrm{~b}$ and $2 \mathrm{c}$, when $k$ is odd, we have

$$
c_{f, 1}^{r, m, n}(k)=\sum_{\substack{a \in \mathbb{Z} / k \mathbb{Z} \\\left((r / 2)^{2}-a(f / 2)^{2}, k\right)=1}}\left(\frac{a}{k}\right)
$$

In either of these cases, we see that the sums vary over congruence classes modulo $k$ which is odd. The multiplicativity of $c_{f, 1}^{r, m}$ now follows from the Chinese remainder theorem and the multiplicative properties of the Legendre symbol. 
Lemma 2.3. Given $r, m$ and $n$, let $i=0$ or 1 and define $\tau$ as follows.

$$
\tau= \begin{cases}2 & \text { if } r \equiv 2 \quad(\bmod 4) ; i=1 \text { and } \operatorname{ord}_{2}(n) \leq \operatorname{ord}_{2}\left(\Delta^{r, m}\right)-2 \\ & \text { and } \operatorname{ord}_{2}(n) \leq 2, \\ \left\lceil\frac{\operatorname{ord}_{2}(n)}{2}\right\rceil+1 \quad & \text { if } r \equiv 2 \quad(\bmod 4) ; i=1 \text { and } \operatorname{ord}_{2}(n) \leq \operatorname{ord}_{2}\left(\Delta^{r, m}\right)-2 \\ & \text { and } \operatorname{ord}_{2}(n)>2, \\ & \text { if } r \equiv 2 \quad(\bmod 4) ; i=1 ; \operatorname{ord}_{2}(n)=\operatorname{ord}_{2}\left(\Delta^{r, m}\right)-1 ; \\ & \text { and } \operatorname{ord}_{2}(n) \text { is odd, } \\ \frac{\operatorname{ord}_{2}\left(\Delta^{r, m}\right)}{2} \quad & \text { if } r \equiv 2 \quad(\bmod 4) ; i=1 ; \operatorname{ord}_{2}(n) \geq \operatorname{ord}_{2}\left(\Delta^{r, m}\right) ; \\ & \text { ord }\left(\Delta^{r, m}\right) \text { is even and } \frac{\Delta^{r, m}}{\operatorname{ord}_{2}\left(\Delta^{r, m}\right)} \equiv 1 \quad(\bmod 4), \\ & \text { if } r \equiv 0 \quad(\bmod 4) \text { and } i=1, \\ 0 & \text { if } r \text { is odd or if } i=0 .\end{cases}
$$

If $f$ is chosen such that $r, m, n$ and $f$ satisfy one of the conditions in lemma 2.1 for $c_{f, i}^{r, m}, n$, and if $q$ is an odd prime, then we have

$$
c_{f, i}^{r, m, n}\left(q^{\alpha}\right)=c_{2^{\tau} q^{\text {ord } q(f)}, i}^{r, m, n}\left(q^{\alpha}\right)
$$

Also, if $r, m, n$ and $f$ satisfy one of conditions $2 a, 2 b$ or $2 c$ of lemma 2.1, then

$$
c_{f, 1}^{r, m, n}\left(2^{\alpha}\right)=c_{2^{\text {ord }_{2}(f), 1}}^{r, m, n}\left(2^{\alpha}\right)
$$

Proof. We will first treat the case when $i=0$ and $r, m, n$ and $f$ satisfy condition (1) of lemma 2.1 Using (6), we have

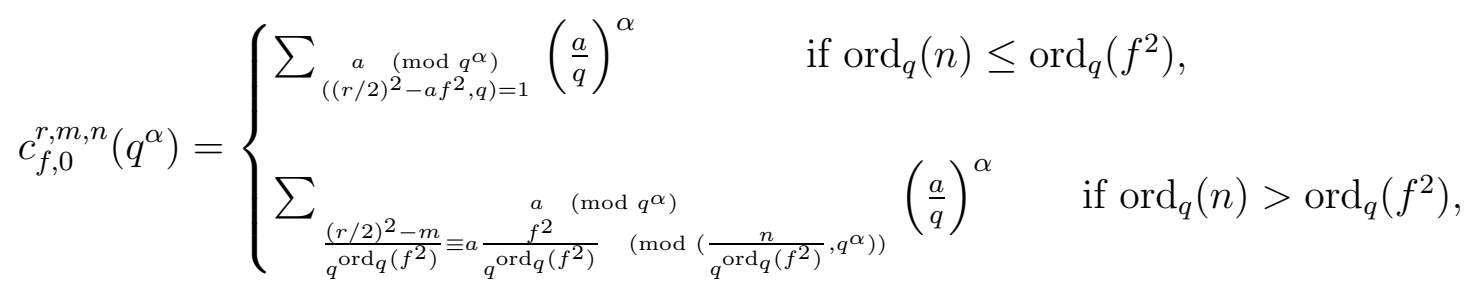

Note that $\frac{f^{2}}{q^{\text {ord } q\left(f^{2}\right)}}$ is a square which is coprime to $q$. Thus making the change of variable $a^{\prime}=$ $a \frac{f^{2}}{q^{\text {ord } q\left(f^{2}\right)}}$, the last sum becomes

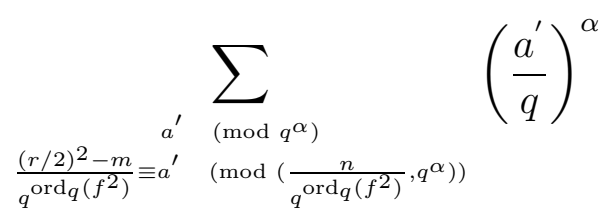

Now combining this with (9), we have

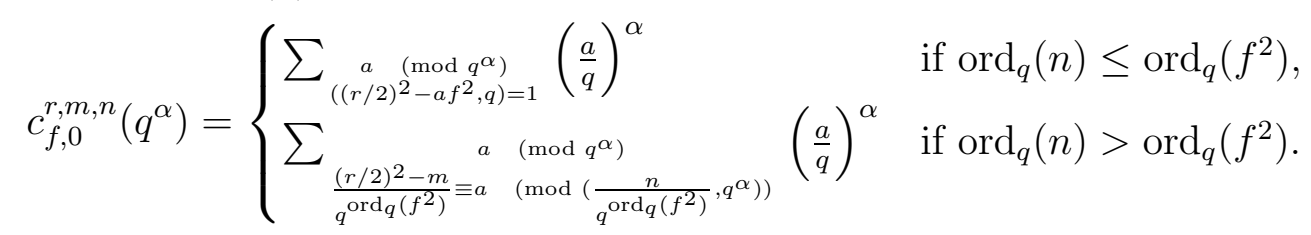


Using this expression one can easily see that $c_{f, 0}^{r, m, n}\left(q^{\alpha}\right)=c_{q^{\text {ord } q(f), 0}}^{r, m, n}\left(q^{\alpha}\right)$, and thus we have proved that the lemma holds in this case.

In all other cases when $q$ is an odd prime, the proof is similar.

For the last assertion, we assume that $r, m, n$ and $f$ satisfy either of conditions $2 \mathrm{~b}$ or $2 \mathrm{c}$ of lemma 2.1. From 5, we have

$$
\begin{aligned}
& c_{f, 1}^{r, m, n}\left(2^{\alpha}\right)=\sum_{\substack{a \equiv\left(\bmod 2^{\alpha+2}\right) \\
a \equiv 1 \\
\frac{(r / 2)^{2}-m}{\left(n,(f / 2)^{2}\right)} \equiv a \frac{(f / 2)^{2}}{\left(n,(f / 2)^{2}\right)}\left(\bmod \left(\frac{n}{\left(n,(f / 2)^{2}\right)}, 2^{\alpha+2}\right)\right)}}\left(\frac{a}{2}\right)^{\alpha} \\
& = \begin{cases}\sum_{\substack{a\left(\bmod \alpha^{\alpha+2}\right) \\
a \equiv 1(\bmod 4)}}\left(\frac{a}{2}\right)^{\alpha} & \text { if } \operatorname{ord}_{2}(n) \leq \operatorname{ord}_{2}\left(f^{2}\right), \\
\sum_{\substack{(r / 2)^{2}-m \\
2^{\operatorname{ord} 2\left(f^{2}\right)-2} \equiv a \frac{f^{2}\left(\bmod 2^{\alpha+2}\right)}{2^{\operatorname{ord}_{2}\left(f^{2}\right)}}\left(\bmod \left(\frac{n}{\left(n,(f / 2)^{2}\right)}, 2^{\alpha+2}\right)\right)}}^{\left(\frac{a}{2}\right)^{\alpha}} & \text { if } \operatorname{ord}_{2}(n) \geq \operatorname{ord}_{2}\left(f^{2}\right)+1 .\end{cases}
\end{aligned}
$$

We note that $\frac{f^{2}}{2^{\text {ord }_{2}\left(f^{2}\right)}}$ is an odd square. Thus, letting $a^{\prime}=a \frac{f^{2}}{2^{\operatorname{ord}_{2}\left(f^{2}\right)}}$ yeilds

$$
c_{f, 1}^{r, m, n}\left(2^{\alpha}\right)= \begin{cases}\sum_{\substack{a\left(\bmod 2^{\alpha+2}\right) \\ a \equiv 1(\bmod 4)}}\left(\frac{a}{2}\right)^{\alpha} & \text { if } \operatorname{ord}_{2}(n) \leq \operatorname{ord}_{2}\left(f^{2}\right), \\ \sum_{\substack{(r / 2)^{2}-m \\ 2^{\operatorname{ord}_{2}\left(f^{2}\right)-2} \equiv a^{\prime}\left(\bmod 2^{\alpha+2}\right)}}^{a_{\left(\bmod \left(\frac{n}{\left(n,(f / 2)^{2}\right)}, 2^{\alpha+2}\right)\right)}^{\prime}}\left(\frac{a^{\prime}}{2}\right)^{\alpha} & \text { if } \operatorname{ord}_{2}(n) \geq \operatorname{ord}_{2}\left(f^{2}\right)+1 .\end{cases}
$$

Using the last expression, one can easily check that $c_{f, 1}^{r, m, n}\left(2^{\alpha}\right)=c_{2^{\text {ord }_{2}(f)}, 1}^{r, m, n}\left(2^{\alpha}\right)$, as desired. In the case that $r, m, n$ and $f$ satisfy condition 2 a of lemma 2.1, the proof is similar.

In order to evaluate the $c_{q^{\beta}, i}^{r, m, n}\left(q^{\alpha}\right),(i=0,1)$, we have the following two lemmas.

Lemma 2.4. Suppose that $q$ is an odd prime and $\alpha>0$. Letting $d=c_{q^{\beta}, 0}^{r, m, n}\left(q^{\alpha}\right)$ when $r$ is even; $(r, q)=1$ and $\left(n, q^{2 \beta}\right) \mid\left((r / 2)^{2}-m\right)$, or letting $d=c_{2^{\tau} q^{\beta}, 1}^{r, m}\left(q^{\alpha}\right)$ when $r, m$ and $n$ satisfy conditions $2 a$, $2 b$ or $2 c$ of lemma 2.1, we have

$$
d= \begin{cases}-\left(\frac{r^{2}}{q}\right) q^{\alpha-1} & \text { if } \beta=0 ; \alpha, \text { odd; } q \backslash n . \\ \left(q-1-\left(\frac{r^{2}}{q}\right)\right) q^{\alpha-1} & \text { if } \beta=0 ; \alpha, \text { even; } q \not n . \\ \frac{q^{\alpha}}{\left(n, q^{\alpha}\right)}\left(\frac{\Delta^{r, m}}{q}\right)^{\alpha} & \text { if } \beta=0 \text { and } q \mid n . \\ 0 & \text { if } \beta>0 ; \alpha, \text { odd and } \operatorname{ord}_{q}(n) \leq 2 \beta . \\ q^{\alpha-1}(q-1) & \text { if } \beta>0 ; \alpha, \text { even and } \operatorname{ord}_{q}(n) \leq 2 \beta . \\ \frac{q^{\alpha}}{\left(\frac{n}{q^{2}}, q^{\alpha}\right)}\left(\frac{\left(\Delta^{r, m}\right) / q^{2 \beta}}{q}\right)^{\alpha} & \text { if } \beta>0 \text { and } \operatorname{ord}_{q}(n)>2 \beta .\end{cases}
$$

Proof. We will prove the lemma for $c_{4 q^{\beta}, 1}^{r, m, n}\left(q^{\alpha}\right)$ where $q$ is an odd prime and where $r, m$ and $n$ satisfy condition $2 \mathrm{~b}$ of lemma 2.1. The proofs for the other cases are similar. From (8) above, we 
have

$$
\begin{aligned}
& c_{4 q^{\beta}, 1}^{r, m, n}\left(q^{\alpha}\right)=\sum_{\substack{a \in \mathbb{Z} / q^{\alpha} Z \\
\left((r /)^{2}-4 a q^{2 \beta}, q\right)=1}}\left(\frac{a}{q}\right)^{\alpha}
\end{aligned}
$$

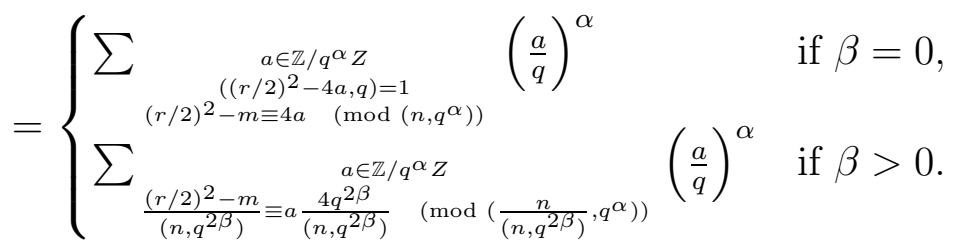

Observe, that when and $q \backslash n$, the second condition of our summation for the case $\beta=0$ is empty. We also note that when $q \mid n$, we have $q \not \mid m$, since $(m, n)=1$. So, the second condition of the summation for the case $\beta=0$ implies the first. With these observations, one can now easily deduce the desired result.

The next lemma allows us to evaluate the $c_{f, 1}^{r, m, n}$ at powers of 2 . The proof is similar to that of the previous lemma and for the sake of brevity we omit it.

Lemma 2.5. (1) If $r$ is odd, then,

$$
c_{1,1}^{r, m, n}\left(2^{\alpha}\right)= \begin{cases}\frac{(-2)^{\alpha}}{2} & \text { if } 4 \not n, \\ \frac{(-2)^{\alpha}}{\left(n, 2^{\alpha}\right)} & \text { if } 4 \mid n,\end{cases}
$$

(2) If $r$ is even and $r, f=2^{\beta}, m$ and $n$ satisfy either of conditions (2b) or (2c) of lemma 2.1, then

$$
c_{2^{\beta}, 1}^{r, m, n}\left(2^{\alpha}\right)= \begin{cases}0 & \text { if } \operatorname{ord}_{2}(n) \leq 2 \beta \text { and } \alpha \text { is odd, } \\ 2^{\alpha} & \text { if } \operatorname{ord}_{2}(n) \leq 2 \beta \text { and } \alpha \text { is even, } \\ \left(\frac{\left((r / 2)^{2}-m\right) / 2^{2 \beta-2}}{2}\right)^{\alpha} \frac{2^{\alpha}}{\left(2^{\text {ord }_{2}(n)-2 \beta}, 2^{\alpha}\right)} & \text { if } \operatorname{ord}_{2}(n) \geq 2 \beta+1 .\end{cases}
$$

Now, let $\kappa(n)$ denote the multiplicative function generated by

$$
\kappa\left(\ell^{\alpha}\right)= \begin{cases}\ell & \text { if } \alpha \text { is odd }, \\ 1 & \text { if } \alpha \text { is even, }\end{cases}
$$

for any prime $\ell$ and any $\alpha>0$. Then we have the following bound.

Lemma 2.6. For all $k, c_{f, i}^{r, m, n}(k) \leq k / \kappa(k)$, where $i=0,1$.

Proof. From lemmas 2.3, 2.4 and 2.5, it follows immideately that for any prime $q$,

$$
\begin{aligned}
c_{f, i}^{r, m, n}\left(q^{\alpha}\right) & \leq \begin{cases}q^{\alpha} & \text { if } \alpha \text { is even }, \\
q^{\alpha-1} & \text { if } \alpha \text { is odd }\end{cases} \\
& =q^{\alpha} / \kappa\left(q^{\alpha}\right) .
\end{aligned}
$$


The lemma now follows from the multiplicativity of $c_{f, i}^{r, m, n}$ and $\kappa$.

We recall the following fact from ([1] Lemma 3.4).

Lemma 2.7. Let $c=\prod_{\ell, \text { prime }}\left(1+\frac{1}{\ell(\sqrt{\ell}-1)}\right)$. Then, $\sum_{k \geq U} \frac{1}{\kappa(k) \phi(k)} \sim \frac{c}{\sqrt{U}}$. In particular, $\sum_{k=1}^{\infty} \frac{1}{\kappa(k) \phi(k)}$ converges.

Thus from lemmas 2.6 and 2.7, we see that $K_{r, m, n}$ is a finite constant.

We rewrite $K_{r, m, n}$ as

$$
K_{r, m, n}=K_{r, m, n}^{0}+K_{r, m, n}^{1}
$$

where

$$
K_{r, m, n}^{0}=\sum_{f=1}^{\infty} \frac{1}{f} \sum_{k=1}^{\infty} \frac{c_{f, 0}^{r, m, n}(k)}{k \phi\left(\left[n, k f^{2}\right]\right)} \quad \text { and } \quad K_{r, m, n}^{1}=\sum_{f=1}^{\infty} \frac{1}{f} \sum_{k=1}^{\infty} \frac{c_{f, 1}^{r, m, n}(k)}{k \phi\left(\left[n, k f^{2}\right]\right)}
$$

Now we compute the constants $K_{r, m, n}^{i}(i=0,1)$. We recall the following identities

$$
\phi(A B)=\phi(A) \phi(B) \frac{(A, B)}{\phi((A, B))},
$$

and therefore, we also have if $B \mid A$,

$$
\phi\left(\frac{A}{B}\right)=\frac{\phi(A) \phi\left(\left(\frac{A}{B}, B\right)\right)}{\phi(B)\left(\frac{A}{B}, B\right)} .
$$

In particular, we can write

$$
\phi\left(\left[n, k f^{2}\right]\right)=\frac{\phi\left(n k f^{2}\right)}{\left(n, k f^{2}\right)} .
$$

Now, we recall for a fixed choice of $r, m$ and $n$, that $f$ must be chosen such that $r, m, n$ and $f$ satisfy the conditions of lemma 2.1 for $c_{f, i}^{r, m, n}(k)$ to be non-zero. We will denote by $S_{i}^{r, m, n}$ the set of $f$ 's which satisfy the conditions of lemma 2.1, and we let $\tau$ be defined as in lemma 2.3. Then, we can write

$$
K_{r, m, n}^{i}=\frac{1}{2^{\tau}} \sum_{\substack{f=1 \\ 2^{\tau} f \in S_{i}^{r, m, n}}}^{\infty} \frac{1}{f \phi\left(2^{2 \tau} n f^{2}\right)} \sum_{k=1}^{\infty} \frac{c_{2^{\tau} f, i}^{r, m, n}(k)\left(n, 2^{2 \tau} k f^{2}\right) \phi\left(\left(2^{2 \tau} n f^{2}, k\right)\right)}{k \phi(k)\left(2^{2 \tau} n f^{2}, k\right)} .
$$

Using lemma 2.2 and the multiplicativity of $\phi$ and letting $(a, b)_{q}:=q^{\operatorname{ord}_{q}((a, b))}$, we can rewrite the inner sum above as,

$$
\prod_{q, \text { prime }}\left(\sum_{j \geq 0} \frac{c_{2^{\tau} f, i}^{r, m, n}\left(q^{j}\right)\left(n, 2^{2 \tau} f^{2} q^{j}\right)_{q} \phi\left(\left(2^{2 \tau} f^{2} n, q^{j}\right)\right)}{q^{j} \phi\left(q^{j}\right)\left(2^{2 \tau} f^{2} n, q^{j}\right)}\right) .
$$

Using lemma 2.3, (24) can be rewritten as 
$(25)$

$$
\begin{aligned}
& \prod_{q \chi f}\left(\sum_{j \geq 0} \frac{c_{2^{\tau}, i}^{r, m}\left(q^{j}\right)\left(n, 2^{2 \tau} q^{j}\right)_{q} \phi\left(\left(2^{2 \tau} n, q^{j}\right)\right)}{q^{j} \phi\left(q^{j}\right)\left(2^{2 \tau} n, q^{j}\right)}\right) \cdot \prod_{q \mid f}\left(\sum_{j \geq 0} \frac{c_{2^{\tau} q^{\text {ord }(f), i}, i}^{r, m, n}\left(q^{j}\right)\left(n, 2^{2 \tau} f^{2} q^{j}\right)_{q} \phi\left(\left(2^{2 \tau} f^{2} n, q^{j}\right)\right)}{q^{j} \phi\left(q^{j}\right)\left(2^{2 \tau} f^{2} n, q^{j}\right)}\right) \\
& =\prod_{q, \text { prime }}\left(\sum_{j \geq 0} \frac{c_{2^{\tau}, i}^{r, m, n}\left(q^{j}\right)\left(n, 2^{2 \tau} q^{j}\right)_{q} \phi\left(\left(2^{2 \tau} n, q^{j}\right)\right)}{q^{j} \phi\left(q^{j}\right)\left(2^{2 \tau} n, q^{j}\right)}\right) \cdot \prod_{q \mid f} \frac{\left(\sum_{j \geq 0} \frac{c_{2^{\tau} q^{r \text { ord } q(f), i}}^{\left(q^{j}\right)\left(n, 2^{2 \tau} f^{2} q^{j}\right)_{q} \phi\left(\left(2^{2 \tau} f^{2} n, q^{j}\right)\right)}}{q^{j} \phi\left(q^{j}\right)\left(2^{2 \tau} f^{2} n, q^{j}\right)}\right)}{\left(\sum_{j \geq 0} \frac{c_{2^{\tau}, i}^{r, m, n}\left(q^{j}\right)\left(n, 2^{2 \tau} q^{j}\right)_{q} \phi\left(\left(2^{2 \tau} n, q^{j}\right)\right)}{q^{j} \phi\left(q^{j}\right)\left(2^{2 \tau} n, q^{j}\right)}\right)} .
\end{aligned}
$$

Now, substituting this last expression back into (23) and using (20), we obtain the following expression for $K_{r, m, n}^{i}$.

$$
\begin{aligned}
\frac{1}{2^{\tau} \phi\left(2^{2 \tau} n\right)} & \prod_{q, \text { prime }}\left(\sum_{j \geq 0} \frac{c_{2^{\tau}, i}^{r, m, n}\left(q^{j}\right)\left(n, 2^{2 \tau} q^{j}\right)_{q} \phi\left(\left(2^{2 \tau} n, q^{j}\right)\right)}{q^{j} \phi\left(q^{j}\right)\left(2^{2 \tau} n, q^{j}\right)}\right) \\
& \cdot \sum_{\substack{f=1 \\
2^{\tau} f \in S_{i}^{r, m, n}}}^{\infty}\left(\frac{\phi\left(\left(2^{2 \tau} n, f^{2}\right)\right)}{f \phi\left(f^{2}\right)\left(2^{2 \tau} n, f^{2}\right)}\right) \cdot \prod_{q \mid f} \frac{\left(\sum_{j \geq 0} \frac{c_{2^{r} q^{\text {ord }}(f), i}\left(q^{j}\right)\left(n, 2^{2 \tau} f^{2} q^{j}\right)_{q} \phi\left(\left(2^{2 \tau} f^{2} n, q^{j}\right)\right)}{q^{j} \phi\left(q^{j}\right)\left(2^{2 \tau} f^{2} n, q^{j}\right)}\right)}{\left(\sum_{j \geq 0} \frac{c_{2^{r}, i}^{r, m}\left(q^{j}\right)\left(n, 2^{2 \tau} q^{j}\right)_{q} \phi\left(\left(2^{2 \tau} n, q^{j}\right)\right)}{q^{j} \phi\left(q^{j}\right)\left(2^{2 \tau} n, q^{j}\right)}\right)} .
\end{aligned}
$$

Now, if $S_{i}^{r, m, n}=\emptyset$, then the above expression is just 0 . So, we will assume for now that $S_{i}^{r, m, n} \neq \emptyset$, and in this case we can rewrite the sum from (26) as a product

$$
\prod_{q, \text { prime }}\left(1+\sum_{\substack{\beta=1 \\ 2^{\tau} q^{\beta} \in S_{i}^{r, m, n}}}^{\infty} \frac{\frac{\phi\left(\left(2^{2 \tau} n, q^{2 \beta}\right)\right)}{q^{\beta} \phi\left(q^{2 \beta}\right)\left(2^{2 \tau} n, q^{2 \beta}\right)}\left(\sum_{j \geq 0} \frac{c_{2^{\tau} q^{\beta}, i}^{r, m, n}\left(q^{j}\right)\left(n, 2^{2 \tau} q^{2 \beta+j}\right)_{q} \phi\left(\left(2^{2 \tau} q^{2 \beta} n, q^{j}\right)\right)}{q^{j} \phi\left(q^{j}\right)\left(2^{2 \tau} q^{2 \beta} n, q^{j}\right)}\right)}{\left(\sum_{j \geq 0} \frac{c_{2 \tau, i}^{r, m, n}\left(q^{j}\right)\left(n, 2^{2 \tau} q^{j}\right)_{q} \phi\left(\left(2^{2 \tau} n, q^{j}\right)\right)}{q^{j} \phi\left(q^{j}\right)\left(2^{2 \tau} n, q^{j}\right)}\right)}\right) .
$$

This allows us to rewrite (26) as 


$$
\begin{aligned}
& \frac{1}{2^{\tau} \phi\left(2^{2 \tau} n\right)} \prod_{\substack{q, \text { odd } \\
q \nmid n}}\left(\begin{array}{l}
\sum_{j \geq 0} \frac{c_{2^{\tau}, i}^{r, m, n}\left(q^{j}\right)}{q^{j} \phi\left(q^{j}\right)} \\
+\sum_{\substack{\beta=1 \\
2^{\tau} q^{\beta} \in S_{i}^{r, m, n}}}^{\infty} \frac{1}{q^{\beta} \phi\left(q^{2 \beta}\right)} \sum_{j \geq 0} \frac{c_{2^{\tau} q^{\beta}, i}^{r, m, n}\left(q^{j}\right) \phi\left(\left(q^{2 \beta}, q^{j}\right)\right)}{q^{j} \phi\left(q^{j}\right)\left(q^{2 \beta}, q^{j}\right)}
\end{array}\right) \\
& \cdot \prod_{\substack{q, \text { odd } \\
q \mid n}}\left(\begin{array}{c}
1+\sum_{j \geq 1} \frac{c_{2^{\tau}, i}^{r, m, n}\left(q^{j}\right)\left(n, q^{j}\right)(q-1)}{q^{j+1} \phi\left(q^{j}\right)} \\
+\sum_{\substack{\beta=1 \\
2^{\tau} q^{\beta} \in S_{i}^{r, m, n}}}^{\infty} \frac{q-1}{q^{\beta+1} \phi\left(q^{2 \beta}\right)} \sum_{j \geq 0} \frac{c_{2^{\tau} q^{\beta}, i}^{r, m, n}\left(q^{j}\right)\left(n, q^{2 \beta+j}\right) \phi\left(\left(q^{2 \beta} n, q^{j}\right)\right)}{q^{j} \phi\left(q^{j}\right)\left(q^{2 \beta} n, q^{j}\right)}
\end{array}\right) \\
& \left(\begin{array}{l}
\left(n, 2^{2 \tau}\right)+\sum_{j \geq 1} \frac{c_{2^{\tau}, i}^{r, m, n}\left(2^{j}\right)\left(n, 2^{2 \tau+j}\right) \phi\left(\left(2^{2 \tau} n, 2^{j}\right)\right)}{2^{j} \phi\left(2^{j}\right)\left(2^{2 \tau} n, 2^{j}\right)} \\
+\sum_{\substack{\beta=1 \\
2^{\tau+\beta} \in S_{i}^{r, m, n}}}^{\infty} \frac{\phi\left(\left(2^{\tau} n, 2^{2 \beta}\right)\right)}{2^{\beta} \phi\left(2^{2 \beta}\right)\left(2^{\tau} n, 2^{2 \beta}\right)} \sum_{j \geq 0} \frac{c_{2^{\tau+\beta}, i}^{r, m, n}\left(2^{j}\right)\left(n, 2^{2 \tau+2 \beta+j}\right) \phi\left(\left(2^{2 \tau+2 \beta} n, 2^{j}\right)\right)}{2^{j} \phi\left(2^{j}\right)\left(2^{2 \tau+2 \beta} n, 2^{j}\right)}
\end{array}\right) .
\end{aligned}
$$

Since, in the first product, $q \not \backslash n$, and since we are assuming that $S_{i}^{r, m, n} \neq \emptyset, 2^{\tau} q^{\beta} \in S_{i}^{r, m, n}$ for all $\beta \geq 1$ if and only if $q \not \gamma$. So using lemma 2.4, the first product in (28) becomes

$$
\prod_{\substack{q, \text { odd } \\ q \nmid n \\ q \nmid r}} \frac{q\left(q^{2}-q-1\right)}{(q+1)(q-1)^{2}} \prod_{\substack{q, \text { odd } \\ q \ngtr n \\ q \mid r}} \frac{q^{2}}{q^{2}-1}
$$

Recalling (3) and (4), and using lemma 2.4 the second product of (28) becomes

$$
\begin{aligned}
\prod_{q \in \mathfrak{Q}_{r, m, n}^{<}} & \left(1+\frac{q\left(\frac{\Delta^{r, m}}{q}\right)+\left(\frac{\Delta^{r, m}}{q}\right)^{2}+\frac{1}{q^{\operatorname{ord} q\left(\Delta^{r, m}\right) / 2}}\left(q \Gamma_{q}+q^{2} \Gamma_{q}^{2}\right)}{q^{2}-1}+\frac{\Gamma_{q}^{2}\left(q^{\left\lfloor\frac{\operatorname{ord}_{q}\left(\Delta^{r, m}\right)-1}{2}\right\rfloor}-1\right)}{q^{\left\lfloor\frac{\operatorname{ord} q\left(\Delta^{r, m}\right)-1}{2}\right\rfloor}(q-1)}\right) \\
& \cdot \prod_{q \in \mathfrak{Q}_{r, m, n}^{\geq}}\left(\frac{q^{\left\lfloor\frac{\operatorname{ord}(n)+1}{2}\right\rfloor}-1}{q^{\left\lfloor\frac{\operatorname{ord} q(n)-1}{2}\right\rfloor}(q-1)}+\frac{q^{\operatorname{ord}_{q}(n)+2}}{q^{3\left\lfloor\frac{\operatorname{ord} q(n)+1}{2}\right\rfloor}\left(q^{2}-1\right)}\right) \cdot \prod_{\substack{q|n \\
q| r \\
q, \text { odd }}}\left(\frac{q\left(q+\left(\frac{-m}{q}\right)\right)}{q^{2}-1}\right)
\end{aligned}
$$


Next, we evaluate the third factor of (28), which we will denote by $T_{i}^{r, m, n}$. Using lemmas 2.1 and 2.5 , we find that

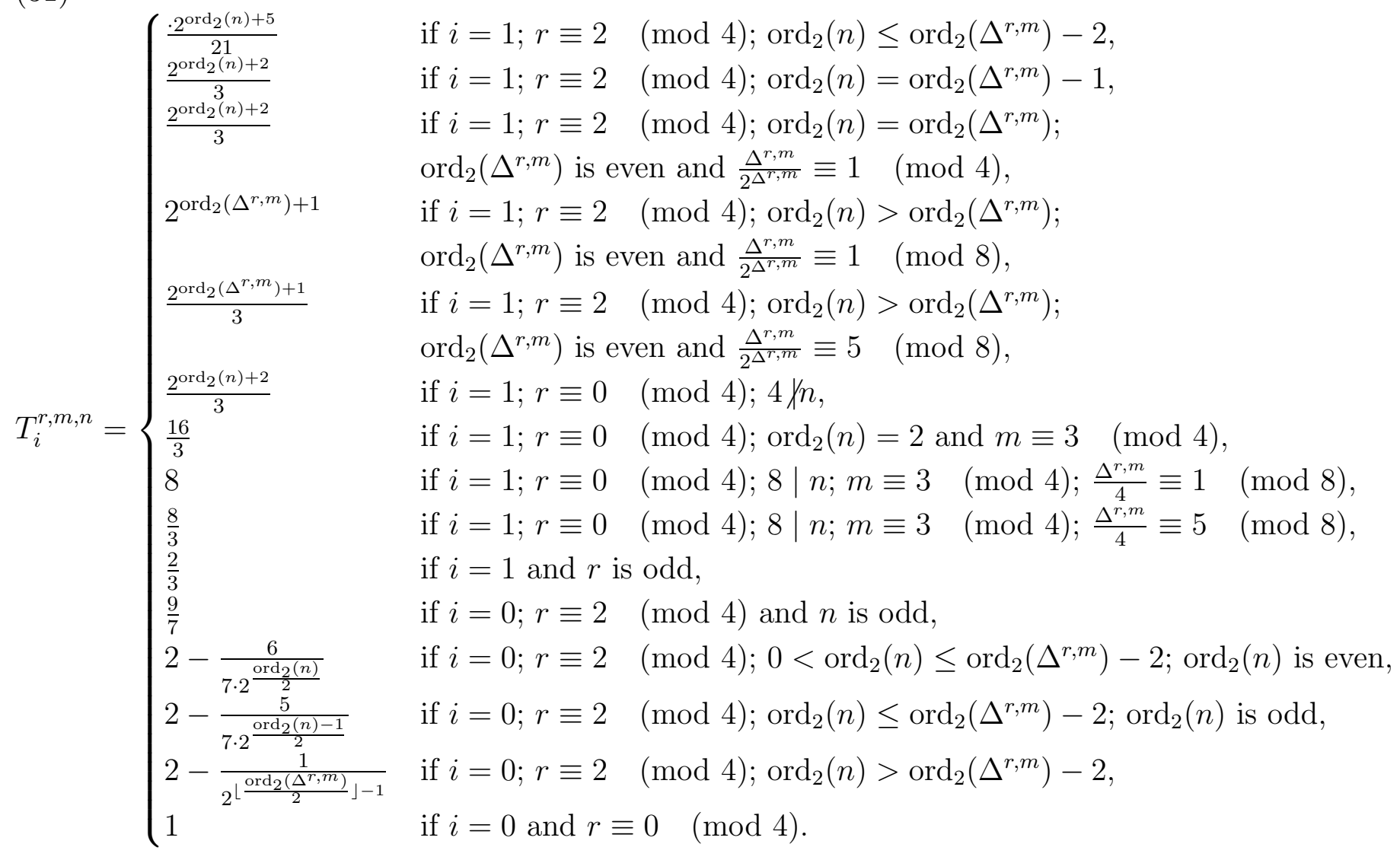

Thus,

$$
\begin{aligned}
& K_{r, m, n}=\left(\frac{T_{0}^{r, m, n}}{\phi(n)}+\frac{T_{1}^{r, m, n}}{2^{\tau} \phi\left(2^{2 \tau} n\right)}\right) \prod_{\substack{q, \text { odd } \\
q \nmid n \\
q \nmid r}} \frac{q\left(q^{2}-q-1\right)}{(q+1)(q-1)^{2}} \prod_{\substack{q, \text { odd } \\
q \nmid n \\
q \mid r}} \frac{q^{2}}{q^{2}-1} \prod_{\substack{q|n \\
q| r}}\left(\frac{q\left(q+\left(\frac{-m}{q}\right)\right)}{q^{2}-1}\right) \\
& \cdot \prod_{q \in \mathfrak{Q}_{r, m, n}^{<}}\left(1+\frac{q\left(\frac{\Delta^{r, m}}{q}\right)+\left(\frac{\Delta^{r, m}}{q}\right)^{2}+\frac{1}{q^{\operatorname{ord} q\left(\Delta^{r, m}\right) / 2}}\left(q \Gamma_{q}+q^{2} \Gamma_{q}^{2}\right)}{q^{2}-1}+\frac{\Gamma_{q}^{2}\left(q^{\left\lfloor\frac{\operatorname{ord} q\left(\Delta^{r, m}\right)-1}{2}\right\rfloor}-1\right)}{q^{\left\lfloor\frac{\operatorname{ord}_{q}\left(\Delta^{r, m}\right)-1}{2}\right\rfloor}(q-1)}\right) \\
& \cdot \prod_{q \in \mathfrak{Q}_{r, m, n}^{\geq}}\left(\frac{q^{\left\lfloor\frac{\operatorname{ord} q(n)+1}{2}\right\rfloor}-1}{q^{\left\lfloor\frac{\operatorname{ord} q(n)-1}{2}\right\rfloor}(q-1)}+\frac{q^{\operatorname{ord}_{q}(n)+2}}{q^{3\left\lfloor\frac{\operatorname{ord} q(n)+1}{2}\right\rfloor}\left(q^{2}-1\right)}\right)
\end{aligned}
$$


Now one can check that $C_{r, m, n}(2)=\phi(n) \cdot\left(\frac{T_{0}^{r, m, n}}{\phi(n)}+\frac{T_{1}^{r, m, n}}{2^{\tau} \phi\left(2^{2 \tau}\right)}\right)$ when $S_{0}^{r, m, n} \cup S_{1}^{r, m, n} \neq \emptyset$ and 0 otherwise. Thus Theorem 1.1 now follows from Proposition 2.1 and from (32).

\section{REFERENCES}

[1] C. David and F. Pappalardi, Average Frobenius distributions of elliptic curves. Internat. Math. Res. Notices (1999) $165-183$.

[2] K. James, "Average Frobenius distributions for elliptic curves with 3-torsion", (preprint).

Department of Mathematical Sciences

Clemson University

BOX 340975

Clemson, SC 29634-0975, USA

kevja@clemson.edu

Department of Mathematical Sciences, Clemson University, BOX 340975 Clemson, SC 29634-0975, USA

E-mail address: kevja@clemson.edu

$U R L:$ http://www.math.clemson.edu/ kevja/ 